\title{
Eduardo Coutinho et la création d'un « espace sonore » dans le documentaire
}

\author{
Gustavo Coura Guimarães*
}

Resumo : Ao longo da sua carreira, Eduardo Coutinho colocou em prática um método de filmagem baseado na experimentação. Passando da ficção ao documentário, do cinema à televisão, o cineasta brasileiro construiu uma técnica de filmagem completamente híbrida, onde as influências de suas diferentes experiências profissionais se encontram. Neste artigo, vamos nos debruçar sobre o papel da palavra filmada nos documentários de Eduardo Coutinho. Nosso objetivo é entender a forma com que este verdadeiro « espaço sonoro » criado entre entrevistador e entrevistados coloca em evidência certos aspectos inerentes à diversidade cultural do Brasil - fato raramente observado em obras de outros cineastas brasileiros.

Palavras-chave : palavra filmada; espaço sonoro ; entrevista; documentário.

Resumen : A lo largo de su carrera, Eduardo Coutinho ha puesto en marcha un método de filmación basado en la experimentación. Pasando de la ficción al documental, del cine a la televisión, el cineasta brasileño ha construido una técnica de filmación completamente híbrida donde se encuentran las influencias de sus diferentes experiencias profesionales. En este artículo, vamos a ver el papel del discurso filmado en los documentales de Eduardo Coutinho. El objetivo es entender cómo este verdadero "espacio sonoro" creado entre cineasta y filmado destaca ciertos aspectos inherentes a la diversidad cultural de Brasil que rara vez observamos en otros directores brasileños. Palabras clave : palabra filmada; espacio sonoro; entrevista documental.

Abstract : During his career, Eduardo Coutinho created a particular way of filming based on experimentation. Passing from fiction to documentary, from cinema to television, the filmmaker has built a totally hybrid method of filming, where all these influences can be observed. In this article, I will analyse the oral speech of Eduardo Coutinho's documentaries. My intention is to understand the way that this real "sound space" created between the director and his witness can reveal some characteristics rooted in the cultural diversity of Brazil rarely observed in the work of other Brazilian filmmakers.

Keywords : recorded word; sound space ; interview ; documentary.

Résumé : Tout au long de sa carrière, Eduardo Coutinho a mis en place une méthode de tournage basée sur l'expérimentation. En passant de la fiction au documentaire, du cinéma à la télévision, le cinéaste brésilien a construit une technique de tournage complétement hybride, où les influences de ses différentes expériences professionnelles se

* Universidade Sorbonne Nouvelle - Paris 3, Escola doutoral "Arts et Médias", Instituto de Pesquisa sobre Cinema e Audiovisual. 75005, Paris, France. E-mail : gcouraguimaraes@gmail.com

Soumission d'article : 10 mai 2017. Notification d'acceptation : 31 juillet 2017.

Doc On-line, n. 22, setembro de 2017, www.doc.ubi.pt, pp. 198-214. 
retrouvent. Dans cet article, nous allons nous pencher sur le rôle de la parole filmée dans les documentaires d'Eduardo Coutinho. L'objectif est de comprendre la façon dont ce véritable « espace sonore » créé entre filmeur et filmés met en évidence certains aspects inhérents à la diversité culturelle du Brésil que nous observons rarement chez d'autres réalisateurs brésiliens.

Mots-clés : mot filmé ; espace sonore ; interview ; documentaire.

\section{L'héritage du journalisme audiovisuel}

Pendant presque 10 ans, la TV Globo a été une sorte de laboratoire pour Eduardo Coutinho. Au sein de cette chaîne, il a pu développer et murir son approche vers l' " autre ». Après avoir quitté de manière précipitée la réalisation du film Un homme à abattre, ${ }^{1}$ Coutinho a fait une pause dans sa carrière de cinéaste. Le contexte de la dictature militaire n'étant pas favorable au métier de réalisateur indépendant, Coutinho a accepté l'invitation qui lui avait été proposée d'intégrer l'équipe du Globo Reporter, une émission hebdomadaire de documentaires pour la télévision. C'est à cette occasion qu'Eduardo Coutinho a pu vérifier ce qui marche et ce qui ne marche pas lors des interviews, c'est-à-dire les inconvénients et les avantages de travailler à la télévision.

Le drame était le suivant : la TV Globo te donne toutes les conditions pour se préparer rapidement, pour préparer une équipe, pour avoir un bon équipement, pour avoir une bonne équipe, de l'argent pour filmer, pour voyager. Et il y a la limitation d'être une télévision : il y a un temps précis, il y a une censure etc. Donc, j'ai une chose et je n'ai pas l'autre. Parce que, si j'étais indépendant, j'aurais toute la liberté de faire le film mais je n'aurais jamais la capacité que l'équipement de la TV Globo a, j'aurais besoin de payer l'équipement, de payer le voyage. [...] Donc, la contradiction est celle-ci : je suis à la TV Globo, en apprenant... non seulement en faisant les films que je pense qui sont importants... Au-delà de tout ça, chaque chose que je fais, en plus de ce qui n'est pas diffusé, soit à cause de la censure, soit parce que je n'ai pas réussi à filmer, m'enrichit profondément par rapport à la réalité du nord-est, qui est ce qui m'intéresse le plus. ${ }^{2}$

1. En portugais, Cabra marcado para morrer.

2. O drama é o seguinte : a Globo te dá todas as condições para se preparar rapidamente, preparar uma equipe, ter um bom equipamento, ter uma boa equipe, dinheiro para filmar, para viajar. E tem a limitação de ser televisão : há um tempo preciso, há censura etc. Então, eu tenho uma coisa e não tenho a outra. Porque, se eu fosse independente, teria toda a liberdade de fazer o filme mas não teria de nenhum jeito a capacidade que tem esse equipamento na Globo, teria que pagar equipamento, pagar a viagem. [...] Então a contradição é esta : eu estou na Globo, aprendendo... não só fazendo os filmes que eu acho que são filmes importantes... Além de tudo, cada coisa que faço, além daquilo que não vai para o ar, seja por censura, seja porque não consegui filmar, me enriquece profundamente em relação à realidade do Nordeste, que é a que mais me interessa. In : Felipe Bragança. Eduardo Coutinho : Encontros. Rio de Janeiro : Beco do Azougue, 2008, p. 199. 
Le croisement entre le journalisme et le cinéma est une des caractéristiques majeures des documentaires d'Eduardo Coutinho. Ces deux domaines se sont imbriqués au cours de la carrière du cinéaste en contribuant à l'élaboration d'une nouvelle forme de représentation de l' « autre » à l'écran. Néanmoins, dans ses films, le réalisateur brésilien ne voulait pas parler de la réalité sociale du Brésil à la place des gens, mais leur donner l'opportunité pour qu'ils s'expriment à leur manière. Cette ambition, pas toujours évidente à mettre en place, est devenue un des éléments les plus représentatifs de son cinéma, puisqu'il le faisait avec une maîtrise rarement observée dans l'histoire du documentaire brésilien. Cette image de soi que les témoins des documentaires d'Eduardo Coutinho ont pu construire d'eux-mêmes au fil des années ressemble beaucoup au concept d' « auto-mise-en-scène » créé par Claudine de France. Autrement dit, ce sont les interviewés qui se mettent en scène en présentant à la caméra une image de soi qu'ils estiment traduire leur essence. Ce rapport privilégié instauré entre filmeur et filmés est le résultat d'une négociation lente e fragile entre les deux instances du discours. Claudine de France appelle cette étape cruciale du tournage comme étant la phase d' «insertion ».

Cette insertion consiste à se faire accepter par les personnes filmées - avec ou sans caméra - et à les convaincre de l'intérêt de collaborer à la réalisation du film comme à l'approfondissement de l'enquête. C'est dire que l'originalité et la réussite de la phase d'insertion tiennent principalement à la qualité morale et psychologique des rapports que parvient à nouer le cinéaste avec les personnes filmées ${ }^{3}$.

Il est important de souligner, avant tout, que cette attention consacrée à la parole des citoyens ordinaires a été viabilisée en grande partie grâce au cinéma direct. La portabilité des équipements techniques, ainsi que la possibilité d'enregistrer le son et l'image simultanément ont contribué à ce que le réalisateur puisse accorder aux personnages de ses films un temps de parole plus étendu. Cependant, le simple fait d'avoir réuni toutes les conditions techniques nécessaires à la réalisation des prises de parole hors des studios ne veut pas dire que le résultat sera forcément réussi. Dans le cadre du cinéma de Coutinho, son expérience à la télévision, ainsi que la mise en place de la méthode des « dispositifs », ${ }^{4}$ jouent un rôle capital dans ce processus.

3. Claudine de France. Cinéma et anthropologie. Paris : Éditions de la Maison des Sciendes de l'Homme, 1989, p. 311.

4. Eduardo Coutinho appelle les « dispositifs » l'ensemble de procédures établies avant le tournage de ses documentaires afin de guider le travail de l'équipe technique sur le terrain. Par exemple, dans le film Jeu de Scène (Jogo de Cena - 2007), le réalisateur a voulu interviewer uniquement des femmes âgées de plus de 18 ans en mélangeant leurs témoignages à des mises-en-scènes d'actrices censées reproduire les témoignages des personnes réelles afin que le spectateur tente de distinguer celle qui raconte sa propre vie de celle qui joue un rôle devant la caméra. 
Tout d'abord, nous aimerions mettre l'accent sur la subtile différence entre le son et la parole. Quand nous parlons de « son », nous faisons référence à toutes sortes de bruits capables d'être enregistrés par des appareils techniques, que nous soyons capables de les distinguer ou pas. En revanche, la «parole» est définie par sa capacité à nous transmettre des informations d'ordre linguistique par le biais de l'expression orale. Dans le cadre du cinéma, son et parole sont présentés au spectateur de façon simultanée. Néanmoins, comme le souligne Michel Chion, l'être humain confère à la parole une priorité instinctive par rapport aux autres composants sonores auxquels il est confronté lorsqu'il est devant l'écran.

Il faut rappeler que la voix possède en effet, comme beaucoup d'autres sons, ce qu'on appelle une image-poids - à savoir la référence à une certaine échelle de puissance, une certaine dimension, une certaine impression référée à l'échelle humaine et qui reste stable même si le son est plus fort ou plus petit selon la distance où il nous parvient. Cette image-poids étant indépendante de l'intensité du son en valeur absolue. ${ }^{5}$

Ainsi, en cherchant instinctivement la compréhension des scènes, le spectateur sélectionne cette « image-poids » qui est censée lui apporter les informations dont il a besoin pour comprendre le déroulement des événements cinématographiques. Cette priorité consacrée à la parole est une des raisons selon lesquelles certains critiques considéraient que le cinéma parlant coupait l'imaginaire du spectateur en lui fournissant le mot.

Il en est de même pour tout espace sonore, vide ou non : qu'il s'y trouve une voix humaine, et infailliblement l'oreille se porte vers elle, l'isolant, et structurant autour d'elle la perception du tout; s'efforçant de décortiquer le son pour en extraire le sens, et toujours cherchant à localiser et si possible à identifier la voix. ${ }^{6}$

Ce bouleversement provoqué par l'arrivée du son a forcément révolutionné le processus de fabrication du récit cinématographique. En fin de compte, l'image photographique n'était plus l'élément principal vers lequel convergeaient tous les messages symboliques transmis par le film. Si, avant, la progression des images était conditionnée à la production du sens, avec l'arrivée du cinéma parlant, les images pourraient être juxtaposées d'une façon désordonnée sans le moindre problème, parce que, de toutes façons, la voix était présente pour pouvoir donner du sens à ce qui, a priori, n'en avait pas.

C'est en s'appuyant sur cette possibilité que le cinéma parlant offrait désormais aux cinéastes, que Coutinho s'est penché sur la mémoire, afin de lui

5. Michel Chion. Le promeneur écoutant : essais d'acoulogie. Paris : Plume/Sacem, 1993, p. 164

6. Ibid., p. 19. 
donner du sens à travers ses représentations dans le documentaire. Aussi désorganisés que les images peuvent l'être, les souvenirs du passé sont gardés dans la mémoire des personnages de ses films de manière dispersée et aléatoire. Les documentaires de Coutinho cherchent donc à donner du sens à cette mosaïque abstraite qui compose la mémoire des citoyens ordinaires. L'outil principal à travers lequel le réalisateur tisse les événements du passé racontés par les personnages en les transformant en film est la parole. Cependant, contrairement à la façon dont les images du passé sont disposées dans la mémoire, ses documentaires ont un propos plus didactique. Dans ce sens, les témoignages recueillis par le réalisateur sont réorganisés d'une telle manière que les faits acquièrent du sens, en étant articulés de manière progressive, afin que le récit soit compréhensible pour le spectateur. Cette opération peut même présenter, en certaines occasions, un anachronisme visuel qui n'échappe pas à la perception du spectateur plus attentif. Voyons dans l'exemple suivant comment cela se produit.

Selon Rodrigues, ${ }^{7}$ le témoignage d'Elizabeth dans Un homme à abattre (1984) illustre de manière conséquente cette remarque. Tout au long de son témoignage, le public voit à l'écran les trois entretiens réalisés avec la veuve de João Pedro Teixeira entrecoupés et partagés en blocs tout au long du récit cinématographique. Il s'agit d'une opération mise en pratique par le réalisateur afin d'apporter de la linéarité au discours d'Elizabeth. Toutefois, cette intervention de la part du cinéaste ne nous empêche pas, pour autant, de remarquer la transformation du personnage depuis le premier entretien jusqu'à la fin du film. Malgré le fait que son témoignage ait été réorganisé par le cinéaste au cours du montage, sa puissance reste, malgré tout, conservée. La critique que certains chercheurs font de cette opération de bricolage réalisée par Coutinho tient compte d'une sorte de dénaturation de la façon dont les souvenirs du passé sont stockés dans la mémoire. Selon eux, le montage réalisé par le réalisateur entrerait en conflit avec la manière dont les souvenirs sont disposés dans la mémoire de ceux qui témoignent. Dans le cas d'Elizabeth, son témoignage a été entrecoupé et réorganisé lors du montage dans le but de donner un sens plus clair et linéaire à son raisonnement.

En raisonnant de manière complètement opposée, le montage réalisé par Coutinho peut être accusé d'être trop didactique. C'est ce qui lui est arrivé lors de la réalisation du film $O$ fio da memória (1991). Pour comprendre la façon dont ce film a été fabriqué, il faut revenir au contexte dans lequel le projet a été conçu. D'après ce que Rodrigues décrit dans sa thèse de doctorat, le film avait

7. Laécio Ricardo de Aquino Rodrigues. A primazia da palavra e o refúgio da memória: o cinema de Eduardo Coutinho. Thèse de Doctorat. Universidade Estadual de Campinas - Unicamp, 2012. 
été commandé par le département de culture du gouvernement de l'état de Rio de Janeiro (1987 - 1991). L'idée était de construire un panorama de la vie des noirs brésiliens pour les commémorations du centenaire de l'abolition de l'esclavage au Brésil. A partir de cette information, nous pouvons déjà identifier le premier obstacle auquel Coutinho a été confronté : représenter la population noire du Brésil dans un film, comme s'il s'agissait d'un groupe homogène et restreint. Ce principe de généraliser des histoires et de les résumer à des formules statiques contrarie toute l'essence du cinéma d'Eduardo Coutinho. Le réalisateur brésilien cherche exactement le contraire dans ses films, puisque, selon lui, l'intérêt de ses documentaires est de montrer la diversité ainsi que les contradictions au sein d'un petit groupe social.

Ensuite, un autre obstacle s'est présenté au cinéaste. Le budget prévu pour la réalisation du film s'est trouvé dépassé. Ainsi, la sortie du documentaire a dû être reportée, afin que l'équipe puisse réunir toute la somme nécessaire à la réalisation du projet. La solution trouvée par les professionnels a été la commercialisation du film vers les chaînes de télévision étrangères. Néanmoins, cette nouvelle tournure prise par le projet a apporté des modifications dramatiques dans la structure du film qui n'ont pas été appréciées par le réalisateur, comme l'extension de la durée à une heure et 55 minutes, ainsi que l'insertion de narrations pour que le sujet soit présenté d'une façon plus didactique aux publics étrangers. Selon la critique, ces exigences commerciales ont abimé le côté esthétique du film, puisque le cinéaste a dû consacrer davantage d'attention aux barrières linguistiques du projet au détriment du côté artistique.

Le troisième problème rencontré par le cinéaste a été l'obligation, pour des raisons commerciales, de réaliser son film en pellicule et pas en vidéo comme il le souhaitait. Ce choix a occasionné de lourdes conséquences lors de la réalisation des entretiens.

L'infime durée des prises de vue en pellicule empêchait les personnages de gagner du temps pour être à l'aise en scène. Souvent, l'entretien finissait précisément quand l'un ou l'autre des interviewés acquérait finalement de la familiarité avec la caméra, ainsi qu'avec l'approche du réalisateur. ${ }^{8}$

Toutes ces péripéties ont reconditionné la construction du documentaire d'Eduardo Coutinho. Au lieu de laisser les interviewés s'exprimer comme ils le souhaitaient et que cette expression authentique soit transmise au spectateur « sans intervention », le cinéaste s'est vu obligé de traduire les symboles de

8. O exíguo tempo de duração das tomadas em película impedia os personagens de ganhar desenvoltura em cena. Não raro, a entrevista findava precisamente quando um ou outro entrevistado finalmente adquiria familiaridade com a câmera e a abordagem do diretor. In : Laécio Ricardo de Aquino Rodrigues. A primazia da palavra e o refúgio da memória : o cinema de Eduardo Coutinho. Thèse de Doctorat. Universidade Estadual de Campinas - Unicamp, 2012, p. 68. 
la culture afro-brésilienne - y compris des symboles linguistiques - afin qu'ils soient compris par le public international. Tous ces obstacles ont retardé la sortie du film de trois ans, et ont contribué au manque de succès du projet lors de sa sortie en salle.

En fonction de tous ces contretemps qui ont conditionné la réalisation de ce film, nous pouvons observer à travers sa structure des passages qui divergent du style des documentaires de Coutinho. Son dispositif a été remplacé par le modèle figé qui conditionne le journalisme audiovisuel dans le but de transformer le projet en un produit commercial. La façon dont les images, les témoignages et la voix off ont été associés démontre cet objectif majeur de formater le film et d'assurer son caractère didactique.

... la vigueur des témoignages individuels se dilue face aux prises de vue vides par l'excès de voix et de descriptions - souvent, certains plans semblent simplement illustrer l'off, ou, de l'autre côté, certains extraits narratifs se limitent à répéter ce que l'image rend évident par soi-même, en promouvant des redondances. Dans l'évaluation de Coutinho, le problème majeur du film est justement l'excès d'explication... ${ }^{9}$

Le choix spécifique de ce documentaire de Coutinho dans le cadre de notre analyse a été fait dans le but de démontrer le poids de la parole au sein de son dispositif. Quand des obstacles se présentent au cours de la réalisation de ses projets, le cinéaste les rend visibles au spectateur, afin qu'il puisse témoigner du processus de fabrication de l'œuvre. Or, dans le documentaire $O$ fio da memoria (1991), les interférences que le film a subies n'ont pas été ressenties au moment du contact entre le réalisateur et les interviewés, mais lors de l'élaboration et de la mise en place du dispositif. En outre, les questions commerciales empêchaient le réalisateur de rendre ces perturbations visibles ou audibles dans le documentaire. Cette interférence est frappante pour ceux qui connaissent l'œuvre du cinéaste et qui constatent que, dans le cas de ce documentaire précisément, il y a quelque chose qui cloche.

Prenons comme exemple la voix off. Comme il s'agit d'un film réalisé en pellicule, Coutinho n'avait pas beaucoup de temps pour faire les entretiens, comme il le faisait normalement lors de ses documentaires filmés en vidéo puis en format numérique. Ainsi, dans ce documentaire, la voix off occupe une place trop importante. Toutefois, pour apporter de l'originalité à la structuration du film et pour sortir un peu du format des reportages de télévision, le réalisateur a pris les mémoires d'un descendant d'une famille d'esclaves

9. O vigor dos depoimentos individuais se dilui frente às tomadas esvaziadas pelo excesso de vozes e de descrições - não raro, alguns planos parecem simplesmente ilustrar o off, ou, por outro lado, alguns trechos narrativos se limitam a repetir o que a imagem torna evidente por si, promovendo redundâncias. Na avaliação de Coutinho, o problema maior do filme é justamente o excesso de explicação. Ibid., p. 70. 
brésilienne comme fil conducteur de l'histoire. Cela permet au cinéaste de recentrer le récit cinématographique, afin qu'il soit moins générique, en faisant en sorte que les différentes prises de vues réalisées avec des communautés afro-brésiliennes variées soient rassemblées autour d'une structure commune. Ainsi, l'histoire de Gabriel Joaquim dos Santos sera le guide du spectateur tout au long de l'expédition proposée par le film vers le Brésil noir.

Coutinho a pris connaissance de son existence grâce au manuscrit de ce citoyen ordinaire, où il enregistrait les faits dont il était témoin. Au moment du tournage, Gabriel était déjà décédé. C'est pour cette raison que ses enregistrements ont été transformés en voix off. Les images qui illustraient les paroles du narrateur étaient des plans de la maison que le personnage avait construit lui-même pour y habiter, ainsi que des plans de son cahier et des images de divinités religieuses dont les descendants des religions africaines sont dévots. Ces images, associées aux descriptions de la voix off, avaient pour but de transporter le spectateur dans le passé, de sorte qu'il soit capable de construire mentalement la personne de Gabriel Joaquim dos Santos et de revivre ses expériences.

J'ai couvert cette maison avec des tuiles le 24 janvier 1943. Je veux beaucoup de respect ici à l'intérieur. Imaginons qu'il s'agit d'une maison religieuse. Ici il y a le nom de Dieu et tout ça. Je dis que le Brésil est une nation sainte, parce que quand Pedro Alvares Cabral a sauté en terre avec les portugais, la première chose qu'ils ont faite c'est une croix. Vous le savez, une croix faite de «pau-brasil ». ${ }^{10}$ Et les portugais à genoux. Et le prêtre « capelão » a célébré une messe (39’05”). ${ }^{11}$

L'aspect curieux de cette procédure mise en place par le réalisateur est que les situations décrites par la voix off ne sont pas vues par le spectateur. Ce sont les symboles associés aux paroles du narrateur qui produisent une image mentale chez le public.

Ce que l'on sait aussi mais que l'on dit moins souvent, c'est que le cinéma parlant consiste aussi à nous montrer une porte fermée ou un rideau opaque, en faisant entendre la voix de quelqu'un supposé être derrière. Ou bien à exposer un espace vide et à faire résonner la voix de quelqu'un supposé être « là », dans le « hic et nunc » de la scène, mais en dehors du cadre. ${ }^{12}$

10. Arbre originaire de la forêt « Mata Atlântica », au Brésil, et qui est devenu un symbole culturel du pays.

11. Eu forrei essa casa com telha em 24 de janeiro de 1943. Eu quero muito respeito aqui dentro. Isso faz de conta que é uma casa religiosa. Aqui tem o nome de Deus por aí e tudo. Eu digo que o Brasil é uma nação santa porque quando Pedro Alvares Cabral saltou em terra com os português, a primeira coisa que fizeram foi uma cruz. A senhora sabe, uma cruz feita de "pau-brasil". E a portuguesada a se ajoelhar. E o padre "capelão" rezou uma missa (39'05"). 30 .

12. Michel Chion. La voix au cinéma. Paris : Editions de l'Etoile, 1982. Réédition 2005, p. 
Contrairement à ce que certains théoriciens pensent du cinéma parlant, l'arrivée de la parole n'a pas empêché que les films fassent appel à l'imaginaire du spectateur pour combler les lacunes présentées par les images. D'autres, comme Béla Bálazs, disent que celui-ci n'est pas le rôle du cinéma, puisqu'il doit, avant tout, signifier à partir de son caractère visuel et non audible. « Au moins aussi nuisible pour le cinéma serait la bonne littérature, parce qu'elle transpose l'action dans une toute autre sphère, une autre dimension, et nous fait perdre la cohérence visuelle des images en nous orientant vers leur cohérence conceptuelle $» .{ }^{13}$

Nous devons admettre que, lorsque le documentaire de Coutinho utilise une telle procédure pour produire une image mentale chez le spectateur, l'essence du cinéma - l'image - est mise de côté dans le but de prioriser le discours oral. C'est lui qui fournit les informations les plus importantes qui guideront le public tout au long de la projection. Les images utilisées pour couvrir la voix off lors de ces séquences, malgré le fait d'être également représentatives, ne sont pas capables de guider le discours filmique, afin que le spectateur soit capable de suivre le déroulement de l'histoire. Ce sont des images qui jouent un rôle plutôt abstrait, voire secondaire, puisqu'elles font appel au répertoire du spectateur, ainsi qu'à son audition, pour pouvoir signifier. La voix qui oriente le discours filmique est placée, de cette manière, à un degré supérieur à celui de l'image. Il s'agit d'une voix qui connaît tout et qui partage ce qu'elle sait avec le spectateur. C'est ce que Chion appelle le principe de l'acousmatique.

L'acousmêtre voit tout, sa parole est comme celle de Dieu : « Nulle créature n'est cachée devant elle. » Celui qui n'est pas dans le champ est le mieux placé pour voir tout ce qui s'y passe; celui que vous ne voyez pas est le mieux placé pour vous voir, c'est du moins le pouvoir que vous lui prêtez. Vous retourneriez-vous pour le surprendre, qu'il pourrait toujours être derrière vous, et ainsi de suite. C'est le fantasme panoptique, de caractère obsessionnel ou paranoïaque, qui est celui d'une maîtrise totale de l'espace par la vue. ${ }^{14}$

Cette voix, connue également comme étant la « voix du savoir », confère au personnage de Gabriel Joaquim dos Santos un certain caractère de réalité. Finalement, il s'agit de la voix qui partage les moments d'intimité du personnage avec le public. Même si la personne de Gabriel a déjà disparu, le spectateur a l'impression de le voir à travers ses mémoires qui sont racontées sous forme de discours oral. Plus qu'avoir l'impression de voir quelqu'un qui n'existe plus, la voix off confère de la légitimité à son discours, puisqu'il s'agit de quelqu'un qui a vécu les expériences décrites par cette voix.

13. Béla Balazs. L'homme visible et l'esprit du cinéma. Traduction : Claude Maillard, Paris : Les éditions Circé, 2010, p. 121.

14. Michel Chion, Ibid., p. 35. 
Selon Chion, ce voyage dans le passé se fait plus facilement à travers le son, parce que, contrairement à l'image, nous ne pouvons pas le maîtriser totalement. Une image projetée à l'écran peut être mise en pause, tandis que le son, quand il s'arrête, est remplacé par le silence.

...beaucoup plus que l'image animée, le son d'une voix, ou bien une ambiance sonore fixés et réécoutés nous réentraînent dans le fil du temps qui passe. Un temps que nous ne pouvons pas maîtriser : il n'y a pas d'arrêt sur le son possible comme il y a des arrêts sur les images, lesquels nous donnent le sentiment de contrôler leur durée. Enregistré, donc emprisonné et conservé, le son des voix et des bruits n'en continue pas moins d'enfermer du temps « à l'état sauvage » (bizarrement seule la musique tonale, parmi toutes les manifestations sonores, peut nous délier du temps réel de son écoute). ${ }^{15}$

Ainsi, c'est comme si le son avait le pouvoir d'emprisonner le temps et de préserver toute sa puissance. Le spectateur, lorsqu'il est confronté aux voix qui l'emmènent vers le passé, n'a donc pas le pouvoir de s'arrêter au milieu de son parcours. Son voyage dans le temps est conditionné à la durée emprisonnée dans les enregistrements sonores.

Dans le cadre de ce film d'Eduardo Coutinho, ce voyage dans le passé est promu par l'association entre la voix off, les témoignages et les extraits de musique qui composent ce que Chion appelle l' «environnement sonore » du documentaire. Cela s'explique grâce à la signification que les images acquièrent lorsqu'elles sont associées au son qui intègre le film. La maison de Gabriel Joaquim dos Santos, par exemple, n'aurait pas eu la même signification si elle avait été montrée sans les descriptions en voix off auxquelles elle était liée. C'est alors le son qui conditionne le message qui sera transmis au spectateur à travers la juxtaposition entre l'image et la parole.

C'est d'ailleurs une des nouveautés qu'amène l'enregistrement de ce qu'on appelle environnement sonore : il fabrique un panorama avec ce qui sur place n'a jamais été perçu comme tel, en réagglomérant des éléments que séparait l'audition directe, et en faisant percevoir de manière plus intense les bruits de fond, dont la présence fonctionne alors sur l'ensemble comme un principe totalisant, unifiant. Le «paysage sonore » est un artefact de l'enregistrement. ${ }^{16}$

Il s'agit d'un environnement sonore créé au sein d'un art qui se revendique comme un art de l'image. Néanmoins, dans le cas spécifique de ce film, l'image n'est pas présentée de manière achevée au spectateur. C'est à lui de la fabriquer mentalement à travers les éléments fournis par le film. C'est une méthode qui nous rappelle le principe de la littérature, qui nous fournit des informations textuelles, afin que nous construisions mentalement les images du

15. Michel Chion. Ibid., p. 144.

16. Ibid., p. 28. 
récit. Dans le cas de ce documentaire, c'est la voix off qui remplace le texte écrit, en s'appuyant sur d'autres composants, comme les images de la maison du personnage central par exemple, pour créer l'univers sur lequel le spectateur doit transiter lors de son voyage dans le passé.

De cette manière, nous considérons que l'image n'est pas totalement méprisée par le réalisateur par rapport à la voix. Malgré le fait que le son ait une place importante au sein de ce documentaire, il est utilisé plutôt pour contextualiser le spectateur et rendre visible les mémoires de Gabriel. En définitive, il n'y a pas assez d'images d'archives capables d'illustrer ce que la voix off raconte. C'est pour cette raison que le texte oral fait appel à l'imaginaire du public pour combler les lacunes qui errent tout au long du récit. Encore une fois, les plans de la maison du personnage illustrent ce propos. Lorsqu'ils sont diffusés, c'est comme si le personnage y était en nous présentant une partie de son intimité.

Dans d'autres circonstances, ce sont les propres témoignages des interviewés qui reconstruisent le passé, en le rendant audible au spectateur. D'ailleurs, il s'agit de la méthode la plus utilisée par Coutinho dans ses documentaires. Les discours des personnages s'enchaînent dans ses films, comme s'il s'agissait de morceaux d'une histoire, dispersés dans l'espace et qui sont rassemblés par le réalisateur sous forme de film. C'est comme si le spectateur se plaçait devant un conteur d'histoires, afin de les revivre par le biais de la force expressive de l'interviewé. Le rôle du réalisateur est de garantir la cohérence du discours, en s'assurant que les témoignages s'accordent entre eux, en fournissant de la progression au récit filmique. C'est, pour cette raison, que la voix off reste toujours présente dans certains de ses documentaires. Sous cet aspect, nous pouvons constater une certaine proximité entre le cinéma de Coutinho et celui de Rouch. Les deux cinéastes ont mis en pratique une méthode de tournage qui privilégie un groupe social minoritaire, afin de mettre en relief la complexité et la diversité existantes au sein de chaque groupe. Dans le cas des documentaires de Jean Rouch, nous observons une particularité. Contrairement à l'époque à laquelle Coutinho a réalisé ses films, dans les années 1940 et 1950, le cinéaste français ne pouvait pas compter sur la vidéo comme méthode d'enregistrement de ses documentaires. Lorsqu'il est parti en Afrique, la caméra utilisée par Rouch exigeait que le réalisateur soit capable de prévoir mentalement la durée de chaque plan, afin que le montage soit réalisé au cours de chaque prise de vue. Ainsi, chaque scène était prévue mentalement avant d'être filmée. La bande sonore devait être rajoutée aux images a posteriori. Lors de cette étape, Rouch avait développé une astuce remarquable, qu'il a mise en pratique dans certains de ses films, comme Moi, un noir (1958). 


\begin{abstract}
Jusqu'au début des années 1960, les appareils 16mm, destinés au marché amateur, ne sont pas conçus pour être associés à un appareil de prise de son. Ce type de caméra, que Rouch utilise lors des tournages en Afrique (Bell \& Howell Filmo 16mm sur le tournage de Maîtres fous en 1954, par exemple) impose une prise de son indépendante des images, problème auquel Rouch répondra de manière totalement innovante en 1958 avec Moi, un noir, en invitant le protagoniste principal à improviser un commentaire lors du premier visionnement des rushs. ${ }^{17}$
\end{abstract}

Nous observons, ainsi, la mise en pratique de deux procédures qui caractérisent le plus le cinéma de Jean Rouch et celui d'Eduardo Coutinho, à savoir la technique du retour des images aux filmés, ainsi que l'attention consacrée à la parole des citoyens ordinaires. Néanmoins, dans le cadre du film Moi, un noir (1958), c'est une question technique - l'impossibilité d'enregistrer l'image et le son simultanément - qui a poussé le réalisateur français à trouver une autre solution pour son problème. De cette manière, c'est comme si la voix du personnage principal du film revivait sa propre histoire lors du visionnage des scènes enregistrées. C'est pour cette raison que nous pouvons considérer que cette procédure mise en pratique par Rouch dialogue d'une façon assez proche avec le cinéma d'Eduardo Coutinho, puisque le réalisateur brésilien utilise aussi des images photographiques ou des extraits filmiques afin d'activer le processus de remémoration des personnages de ses documentaires. Toutefois, dans le cadre des films du réalisateur brésilien, cette méthode a le but de réveiller les souvenirs du passé de ceux qui témoignent devant sa caméra à l'instant précis de l'établissement du contact entre filmeur et filmé. Ainsi, leurs discours, enregistrés lors de cette remémoration, portent toute la puissance émotionnelle ressentie par les personnages lorsqu'ils se souviennent de leurs histoires passées.

Nous pouvons donc considérer que la méthode de Jean Rouch est, même de manière intuitive, à l'origine du dispositif cinématographique mis en place par Coutinho au cours de sa carrière. $\mathrm{Si}$, pour des raisons techniques, le réalisateur français ne pouvait pas aller plus loin dans sa démarche, le cinéaste brésilien a su s'inspirer de la méthode mise en pratique par Rouch en l'adaptant au contexte social du Brésil, ainsi qu'aux appareils techniques capables d'enregistrer le son et l'image simultanément dont il disposait à l'époque. C'est pour cette raison que nous considérons que les images enregistrées par les deux cinéastes communiquent sous plusieurs aspects. Les deux ont su exploiter les potentialités du son, afin d'enrichir leurs représentations cinématographiques, chacun en son temps et dans des contextes très spécifiques. Cependant, cela

17. Séverine Graff. Techniques légères et cinéastes du direct : un cas de "Rouchéole». Colloque International Jean Rouch, 14-20 nov. 2009, p. 3. 
n'empêche pas que les images voyagent dans le temps et qu'elles se parlent entre elles. C'est le principe de la migration des images dont nous parle Jacques Aumont ${ }^{18}$ et que nous appliquons aux spécificités de notre objet. Dans le cadre de notre recherche, c'est comme si Rouch était à l'origine de la technique développée par Coutinho tout au long de son parcours, en servant d'inspiration pour que le réalisateur brésilien s'aperçoive de toute la puissance que la parole peut apporter aux représentations ethno-cinématographiques. La technologie de la vidéo a été le coup de pouce qui a rendu possible l'adaptation de la méthode rouchienne au contexte dans lequel le réalisateur brésilien a réalisé ses documentaires. Nous ne défendons pas, pour autant, l'hypothèse qu'Eduardo Coutinho soit une sorte de «Jean Rouch Brésilien ». Cependant, comme il s'agit d'un réalisateur qui avait une grande connaissance dans le domaine du cinéma, notamment du cinéma documentaire, nous ne pouvons pas amoindrir le rôle que les films de Jean Rouch ont probablement joué dans la conception des « dispositifs » qu'il a mis en pratique par la suite tout au long de sa carrière.

\section{Le rôle de la parole filmée au sein du contexte culturel brésilien}

Pour mieux comprendre la dynamique sociale brésilienne actuelle, il faut remonter à l'époque de la colonisation du pays, en 1500. Lors de l'arrivée des portugais au Brésil, le contact entre la population indienne et européenne ne s'est pas établi de manière amicale. Peu après avoir débarqué sur le sol brésilien, les portugais ont initié un processus d'évangélisation des indiens dans le but de leur imposer la religion catholique ainsi que les règles de l'esclavage auxquelles ils seraient désormais soumis. Il s'agit du premier choc culturel qui a eu lieu au Brésil entre deux populations complétement distinctes.

Plus tard, la main d'œuvre indienne étant insuffisante pour l'exploration de l'ensemble du territoire brésilien, les portugais ont fait venir des esclaves noirs de l'Afrique. Le but était d'explorer l'intérieur du pays à la recherche de richesses naturelles qui seraient ensuite commercialisées en Europe.

Ce processus lent, douloureux et sanglant a marqué un chapitre important de l'histoire du Brésil. Pendant plus de 300 ans, l'exploitation de l'homme par l'homme a dessiné le tissu social brésilien dont les traces sont toujours visibles dans la société actuelle. Les populations indienne et noire, reléguées pendant plusieurs siècles au rez-de-chaussée de la pyramide sociale, n'ont jamais pu sortir complétement de cette relation d'esclavage à laquelle elles ont été condamnées depuis le début de la colonisation. Même la loi d'abolition de

18. Colloque « Décrire, construire le cinéma », réalisé les 17 et 18 mars 2012 à l'Université Paris Diderot - Paris 7. 
l'esclavage, promulguée en 1888, n'a pas donné les moyens nécessaires pour que les esclaves du Brésil soient réellement libres et indépendants.

De cette manière, au fil des années, l'écart entre les blancs et les exesclaves brésiliens et leurs descendants n'a cessé d'augmenter. L'ascension sociale se montrait de plus en plus rare et les inégalités entre les deux couches de la population sont devenues encore plus importantes.

A Rio de Janeiro, cette disparité entre les côtés riche et pauvre du Brésil se traduit par l'apparition des «favelas », situées juste à côté des quartiers les plus bourgeois de la ville. Ce mode d'organisation sociale a été instauré dans la ville de Rio de Janeiro prioritairement pendant le mandat du maire Francisco Pereira Passos, à partir de 1902. Son objectif était de moderniser le centre-ville en lui donnant l'air d'une ville européenne. Pereira Passos s'est inspiré de la ville de Paris, où il a fait ses études à la fin des années cinquante. Ainsi, pour mener les réformes prévues dans le cadre de son projet, il a dû expulser la population qui avait occupé le centre-ville et qui vivait dans des conditions extrêmement précaires. Son but était de moderniser la façade des immeubles et de prioriser l'accès au centre-ville aux membres des classes les plus aisées. Pour y parvenir, les anciens habitants des immeubles précaires du centre-ville de Rio ont dû se reloger de manière précipitée et désordonnée dans les collines qui entourent la ville. Sans aucune aide de l'Etat, ces constructions ont été financées par les habitants eux-mêmes. C'est ainsi que, au fil des années, les "favelas » ont dessiné le paysage de Rio de Janeiro. Néanmoins, les conditions précaires dans lesquelles les habitants y vivaient n'ont pas été changées. Le manque d'infrastructure est resté le même et l'exposition à des maladies demeurait un des problèmes majeurs auxquels ils devaient faire face.

Dans cette perspective, la verticalisation des relations entre les classes sociales au sein de la société brésilienne est restée inchangée depuis l'époque de la colonisation. La séparation entre les côtés riche et pauvre de la population a toujours conditionné l'occupation du territoire. La ville de Rio de Janeiro est un des exemples les plus explicites de la façon dont la dynamique sociale brésilienne a été déterminée à partir des relations de domination qui se sont créées dans le pays depuis l'arrivée des portugais.

C'est exactement cette fracture sociale que le cinéaste Eduardo Coutinho a voulu explorer dans ses documentaires. A partir de son expérience à la télévision, le réalisateur a pu observer que la population marginalisée du Brésil avait une grande richesse culturelle et historique que le grand public ignorait la plupart du temps. Il a donc décidé d'explorer cette partie oubliée du territoire brésilien et de montrer au public toute la complexité inhérente à cet univers. 
Si d'un côté les médias traditionnels parlent souvent de cette partie de la population brésilienne de manière superficielle et stéréotypée, de l'autre côté Coutinho met en place une méthode de tournage qui consiste à donner aux interviewés un temps de parole plus étendu pour qu'ils puissent s'exprimer à leur manière. Libre des préjugés et des caricatures qui parfois polluent l'image des habitants des «favelas », dans les documentaires de Coutinho nous découvrons un autre visage de cette population. Ses films révèlent un véritable exercice de découverte de l'« autre » par le biais de la parole filmée. A partir d'un minimalisme esthétique remarquable, le cinéaste brésilien arrive à créer une sorte d' « espace sonore », où les témoins livrent leurs mémoires du passé lorsqu'ils sont placés devant la caméra. Le résultat de cette confrontation entre filmeur et filmés est une interaction authentique, car, selon Coutinho, la fraîcheur de la première rencontre est essentielle pour que les interviewés puissent livrer leurs souvenirs du passé.

Coutinho dispose d'informations de ses personnages, un «type de munition » qui, dans les mains d'un cinéaste inexpérimenté, convertit la rencontre en discussion dirigée, prévisible. Dans de tels films, Coutinho intercepte ses interlocuteurs sur des situations et des histoires déjà recueillies par l'équipe de recherche et qui lui sont retransmises (il y a donc une direction); néanmoins, compte tenu de la fraîcheur de la rencontre entre le réalisateur et les personnages, la capacité d'agencement du cinéaste (qui suscite l'exhibitionnisme et la dimension fabulatrice des sujets en scène) et l'articulation ouverte des questions, favorable au « délire », cette rencontre, presque toujours, gagne des contours surprenants et inattendus pour les deux pôles du processus communicatif (personnages, cinéaste et spectateurs), en « en fuyant » les directions suggérées par la recherche préalable.

Ainsi, à partir de cette méthode de tournage, le cinéaste brésilien met en évidence les nuances d'une population métissée et hétérogène qui ne se restreint pas aux stéréotypes souvent véhiculés par les médias traditionnels. Par le biais du « dispositif» mis en place dans chaque documentaire, Coutinho creuse cet « espace sonore » créé entre lui et l'interviewé afin de montrer au spectateur le « lieu de parole » de ceux qui témoignent.

Les théories modernes de l'énonciation nous forcent à admettre que l'énonciation provient toujours de quelque part. Elle ne peut pas ne pas être située. Elle ne peut pas ne pas être positionnée. Elle est toujours positionnée dans un discours. Et c'est quand un discours oublie qu'il est situé qu'il en vient à prétendre parler au nom de tous les autres. ${ }^{19}$

Nous pouvons donc observer que la parole filmée occupe une place privilégiée au sein du cinéma d'Eduardo Coutinho. Tout au long de sa carrière, le

19. Stuart Hall. Identités et cultures 2 : politiques des différences. Paris : Editions Amsterdam, 2013, p. 48. 
réalisateur brésilien a appris à travailler avec le discours oral des interviewés avec une telle aisance grâce aux différentes expériences professionnelles qu'il a eues. En montrant au spectateur la façon dont le contact entre filmeur et filmés est construit, Coutinho dévoile non seulement les souvenirs du passé stockés dans la mémoire de ses interviewés, mais également les contraintes inhérentes à cette prise de contact, sans pour autant vouloir parler de leur vie à leur place. La mise en valeur de ce lien fragile entre les deux instances du discours est une des marques identitaires les plus éloquentes de son cinéma. Au sein d'un art réputé comme étant l'art de l'image en mouvement, les documentaires d'Eduardo Coutinho nous montrent que la parole filmée peut se révéler extrêmement puissante. Il suffit de savoir la manipuler avec la dextérité nécessaire afin de montrer au spectateur que les combinaisons entre image et son sont multiples, puisque, dans le cadre du cinéma d'Eduardo Coutinho, elles ne cessent de se réinventer.

\section{Références bibliographiques}

Agamben, G. (2007). Qu'est-ce qu'un dispositif. Paris : Editions Payot \& Rivages.

Andrade, O. de (2011). A utopia antropofágica, 4ème édition. São Paulo : Globo.

Balázs, B. (2010). L’homme visible et l'esprit du cinéma. Traduction : C. Maillard. Paris : Les éditions Circé.

Bezerra, C. (2014). A personagem no documentário de Eduardo Coutinho. Campinas : Papirus.

Bragança, F. (2008). Eduardo Coutinho : encontros. Rio de Janeiro : Beco do Azougue.

Chion, M. (2005). La voix au cinéma. Paris : Editions de l'Etoile, 1982. Réédition.

Chion, M. (1993). Le promeneur écoutant : essais d'acoulogie. Paris : Plume/ Sacem.

Comolli, J.-L. (2004). Voir et pouvoir, l'innocence perdue : cinéma, télévision, fiction, documentaire. Paris : Editions Verdier.

France, C. de (1989). Cinéma et anthropologie, (p. 311). Paris : Éditions de la Maison des Sciences de l'Homme.

Graff, S. (2009). Techniques légères et cinéastes du direct : un cas de «Rouchéole». Colloque International Jean Rouch, 14-20 nov. 
Hall, S. (2013). Identités et cultures 2 : politiques des différences. Paris : Editions Amsterdam.

Lins, C. (2007). O documentário de Eduardo Coutinho : televisão, cinema e vídeo. Rio de Janeiro : Zahar.

Rodrigues, L. R. de A. (2012). A primazia da palavra e o refúgio da memória : o cinema de Eduardo Coutinho. Thèse de Doctorat. Universidade Estadual de Campinas - Unicamp.

Odin, R. (2011). Les espaces de communication : introduction à la sémiopragmatique. Grenoble: Presses Universitaires de Grenoble. 